\title{
EL CAMBIO CLIMÁTICO Y SUS IMPLICACIONES EN LA SALUD HUMANA
}

\author{
CLIMATE CHANGE AND ITS IMPLICATIONS \\ FOR HUMAN HEALTH
}

\author{
Tania Angélica Cuadros Cagua*
}

Resumen

El objetivo de este artículo es analizar, a través de la revisión de literatura, los efectos del cambio climático sobre la salud de las personas. Para ello se hizo una revisión de la documentación científica publicada sobre los efectos del cambio climático en la salud humana y se señalan algunos de los efectos que los fenómenos secundarios al cambio climático, tales como las temperaturas extremas, las olas de calor, los cambios en la calidad del aire o del agua tienen sobre la morbimortalidad de la población. Una de las conclusiones del estudio es que los fenómenos secundarios al cambio climático tienen efectos directos e indirectos sobre la salud humana, lo que hace que las repercusiones sean diversas y se solapen unas con otras, al tiempo que algunas serán difícilmente reversibles en años o decenios.

* Abogada y enfermera, Especialista en Auditoría en Salud y en Gerencia en Salud Ocupacional. Ciencias Médicas y de la Salud -- Ciencias de la Salud -- Ciencias del Cuidado de la Salud y Servicios (Administración de Hospitales, Financiamiento)

\section{Palabras clave}

Cambio climático - salud humana - efectos directos e indirectos

\section{Abstract}

The purpose of this article is to analyze, employing a literature review, the effects of climate change on the health of people. To this end, we reviewed the scientific literature published on the consequences of climate change on human health and pointed out some of the effects secondary phenomena of climate change -such as extreme temperatures, heat waves, changes in the quality of air or water- have on the morbidity/mortality of the population. One of the conclusions of the study is that phenomena secondary to climate change have direct and indirect effects on human health, which gives rise to diverse and overlapping repercussions, with some others that will be difficult to reverse in years or decades.

Keywords:

Climate change - human health - direct and indirect effects 


\section{Introducción}

El ser humano, en su desarrollo como especie biológica, ha estado estrechamente vinculado a las variaciones climáticas que condicionan su evolución. Los elementos que determinan la salud de las poblaciones son muy diversos e interactúan de modo complejo, pero, sin dudas, los estudios evidencian cada vez más que las alteraciones climáticas y del medio ambiente tienen repercusiones en la salud humana (Patz et al., 2000; Iniesta, Ríos, Fernández y Barbado, 2008).

El presente artículo analiza, a través de la revisión de literatura, los efectos que los fenómenos secundarios al cambio climático, tales como las temperaturas extremas, las olas de calor y los cambios en la calidad del aire o del agua tienen sobre la morbimortalidad de la población.

Este documento es un aporte más al conocimiento que se tiene sobre las interdependencias de la especie humana, su evolución y la manera en que el cambio climático afecta $o$ puede llegar a afectar la salud de las personas. Así mismo, contribuye a entender que la complejidad y multicausalidad de las alteraciones de la salud humana vinculadas con el cambio climático, además de constituir un reto para los sistemas de salud, requiere que en la búsqueda de soluciones se emplee el enfoque inter y transdisciplinar, para dotarla de visiones y marcos teóricos que puedan aportar nuevos elementos conceptuales en el abordaje, la comprensión y el manejo de la dinámica real, integral, holística y sistémica de los problemas de salud humana.

\section{Metodología}

Para este artículo, la búsqueda bibliográfica partió en primer lugar de la recomendación de la Organización Mundial de la Salud (OMS) respecto de la relevancia que tiene para la salud pública informarse acerca de las amenazas para la salud de la población relacionadas con la interacción de los determinantes ambientales y sociales de la salud, en el marco del cambio climático. El segundo factor motivador fue la consulta sobre los temas de salud que recomienda investigar la Convocatoria para Proyectos de Ciencia, Tecnología e Innovación en Salud - 2016 de Colciencias y el Ministerio de Salud y Protección Social, entre los que figura la salud ambiental, las enfermedades infecciosas transmitidas por vectores y las zoonosis, las que a su vez están asociadas a los efectos del cambio climático.

Se consultaron 150 de las más recientes investigaciones, informes institucionales de los organismos de la Organización de las Naciones Unidas (ONU [Panel Intergubernamental de Expertos sobre el Cambio Climático (IPCC, por sus siglas en inglés), OMS, Organización Internacional Para Las Migraciones (OIM)]) y National Institutes of Health [NIH] sobre el cambio climático y sus afectaciones a la salud humana. La búsqueda se realizó en bases de datos de medicina como Medline, Biblioteca Virtual de Salud (BVS), ScienceDirect, Pubmed, Scielo y Redalyc. Los tópicos por los cuales se depuraron y seleccionaron las 53 publicaciones para elaborar el presente artículo fueron el título, el(los) autor(es), el resumen, los objetivos y las conclusiones. La descripción de las afectaciones a la salud humana se realizó con base en las categorías (comunes) de consecuencias establecidas por la OMS y el NIH, sin que el orden en que aparecen indique su priorización. Es de resaltar que tampoco se tuvo en cuenta para su reseña, la clasificación de los efectos que diversos autores e instituciones han formulado en estos años de interés sobre el tema. 


\section{El cambio climático}

El sistema climático mundial es parte integrante de los complejos procesos que mantienen la vida, pero el incremento progresivo de la temperatura terrestre está produciendo una interferencia en sus ciclos, y la actividad económico-social es la principal causa (McMichael, 2003; IPCC, 2014).

El término cambio climático denota toda variación del clima (del valor medio o sus propiedades) a lo largo del tiempo (decenios o periodos más largos). El cambio climático también obedece a causas naturales, tales como modulaciones de los ciclos solares, erupciones volcánicas, o a la actividad humana, que alteran la composición de la atmósfera global y se suman a la variabilidad natural del clima (IPCC, 2007, 2014).

El cambio climático conlleva variaciones o degradación de los ecosistemas naturales y antropogénicos como resultado de una lenta y compleja interacción de diversos efectores de cambio, que son a la vez causas y consecuencias (directas e indirectas) y, lejos de ser independientes, llevan tanto a sinergias ocasionales como a potenciaciones del impacto de sus efectos sobre la salud humana (Convención Marco de las Naciones Unidas sobre el Cambio Climático, 1992; Beniston, 2002; Calvo, 2008; Sánchez et al., 2009; Lozano, 2016).

\section{Repercusiones del cambio climático en la salud humana}

La relación causa-efecto del cambio climático para determinadas enfermedades humanas es conocida desde la Antigüedad (OMS, 2003; Iniesta et al., 2008) y, aunque nunca se ha priorizado su estudio (Sánchez, 2016), solo en los últimos decenios y ante las rotundas evidencias disponibles, se incluye en amplios espacios de discusión y decisión en todos los niveles (Calvo, 2008; IPCC, 2014).

No todos los posibles efectos atribuidos al cambio climático son negativos, pues el calentamiento mundial puede tener algunos efectos beneficiosos localizados, como una menor morbimortalidad en invierno por causa cardiovascular y un aumento de la producción de alimentos en determinadas zonas (IPCC, 2014), pero, en general, las repercusiones en la salud serán probablemente muy adversas (OMS, 2016a; Ochoa et al., 2015).

Si se tiene en cuenta que el proceso salud-enfermedad es multidimensional, las alteraciones para la salud humana atribuibles al cambio climático y distinguibles de otras influencias son de difícil demostración, en especial si se hace foco en un único factor causal (Beniston, 2002; Burstein, 2016), por lo que se requiere la interpretación e interrelación multi y transdisciplinar (Berberian y Rosanovaa, 2012).

El cambio climático es una creciente amenaza para la salud humana, porque aumenta la probabilidad de impactos graves, generalizados e irreversibles en los determinantes sociales y medioambientales de la salud: agua potable, aire limpio, alimentos suficientes y abrigo adecuado - vivienda segura- (IPCC, 2001, 2007, 2014; OMS, 2008; Costello et al., 2009; Ponce y Cantú, 2012; OMS, 2016b), en especial para los grupos con mayor vulnerabilidad (OMS, 2003; Haines y Patz, 2004; Ballester y Moreno, 2006; Iniesta et al., 2008; Calvo, 2008; Berberian y Rosanovaa, 2012) asociada a otros determinantes sociales como los demográficos, los socioeconómicos, los políticos y los culturales (Sánchez, 2016).

En este orden de ideas, y teniendo en cuenta que las repercusiones del cambio climático sobre la salud humana son diversas, 
en la tabla 1 se presentan las categorías en las cuales, tradicionalmente, se han clasificado los efectos del cambio climático.

A continuación, se describen brevemente los impactos del cambio climático en los sistemas naturales y sus efectos sobre los sistemas humanos, como la salud, la seguridad alimentaria y la seguridad de las condiciones sociales, tomando como base las categorías (comunes) de consecuencias establecidas por la OMS (2008) y el NIH (2010).

Clasificación de los efectos del cambio climático sobre la salud humana

Autor/institución Clasificación Patz et al. (2000), Beniston (2002), Orellana y Navarro (2010), Berberian y Rosanovaa (2012) y Ochoa et al. (2015)

Directos: por acción del calor o el frío, como el calor excesivo y las olas de calor.

Indirectos: provocados por el calor o el frío en otros organismos, sistemas geoquímicos (sistemas naturales de lluvias, cuerpos de agua naturales y artificiales) o en las edificaciones. Por ejemplo: cambios en la calidad de las aguas y alimentos, alteración de los bosques que estimulan la migración, la distribución y la proliferación de vectores transmisores de agentes patógenos. Butler, Corvalan y Hillel, (2005), Butler y Harley (2010), McMichael (2014) y Useros (2014)

Primarios: son de efecto inmediato y evidente relación causa-efecto, asociados a peligros físicos por la acción directa de la exposición climática ambiental, tales como: el calor excesivo y las olas de calor, inundaciones, tormentas, tornados e incendios forestales.

Secundarios: son el resultado de vías menos directas, al ser menos rápidos y evidentes, lo que hace que su causalidad resulte más problemática.

Implican interrupciones o cambios en los ecosistemas y las relaciones entre las especies, entre ellos: las relaciones entre los rendimientos de la cosecha, los agentes biológicos productores y transmisores de enfermedades infecciosas, la distribución de los alérgenos, la concentración de contaminantes atmosféricos, incremento en los problemas en la salud mental (depresión, ansiedad y trastorno por estrés postraumático) por los desastres relacionados con el cambio climático.

Terciarios: representan los riesgos más graves y devastadores para la salud física y mental, producto de las decisiones políticas, trastornos sociales y económicos por pérdida o cambios de empleos, situaciones de conflicto impulsado por migraciones masivas de carácter forzoso y escasez de recursos relacionados con el cambio climático (agua, alimentos, tierra habitable).

IPCC (2014) Mediados por el ecosistema: surgen de un desequilibrio en las relaciones entre varios factores, además del clima. En ellas se incluyen las enfermedades transmitidas por vectores, infecciones transmitidas por agua y alimentos y calidad del aire.

Directos del clima sobre la salud: consecuencias directas del clima, entre ellos efectos del calor y del frío, inundaciones, tormentas y radiación ultravioleta

Mediados por instituciones humanas: directamente atribuibles a la actividad humana. También tienden a ser más crónicas y difíciles de medir (nutrición, salud ocupacional, salud mental violencia y conflicto).

Efectos del cambio climático sobre la salud humana 


\section{Evento Efectos}

\section{Altas temperaturas terrestres}

Elevación de tasas de morbimortalidad (Ledrans et al., 2004; Mendoza, Martínez, Contreras y Fajardo, 2012), en especial para las personas de mayor riesgo, como adultos mayores, niños, mujeres embarazadas y personas con patologías crónicas cardiovasculares o respiratorias (Huynen, Martens, Schram, Weijenberg y Kunst, 2001; Más, 2010).

El aumento en la humedad relativa empeora la incomodidad e incrementa el riesgo para la salud al evitar la evaporación del sudor, impidiendo que el cuerpo se enfríe (Sánchez, 2016).

Probable aumento de ozono atmosférico al nivel del suelo (Noyes et al. 2009).

Aceleran el inicio o prolongación de la estación del polen (primavera), lo cual favorece la presentación de ataques de asma y alergias (Petersson, Marrero y Taboada, 2010).

\section{Fenómenos meteorológicos extremos}

Desastres naturales por perturbaciones de los regímenes pluviales: huracanes, tormentas, ciclones, inundaciones o sequías (dependiendo de la región) (Iniesta et al., 2008; Peterson et al., 2010)

Incremento de la mortalidad y la morbilidad por lesiones físicas, psíquicas, económicas y sociales.

Desplazamientos de regiones (ecológicas y agrícolas) y de población, los cuales generan problemas como hacinamiento y falta de refugio (OIM, 2007; Jiménez y Soledad, 2011).

Escasez y deterioro de la calidad del agua para consumo humano, por almacenamien- to inadecuado y contaminación, lo cual hace que aumenten los brotes de enfermedades diarreicas, en especial si se han visto dañados o destruidos los sistemas de abastecimiento de agua y manejo de residuos líquidos, por lo que imperan condiciones de saneamiento deficientes (OMS, 2008; Feo, 2009; Ochoa et al, 2015; Hijar, Bonilla, Munayco, Gutiérrez y Ramos, 2016).

Afectación de la seguridad alimentaria debido a cambios en los niveles de producción de alimentos, lo cual ocasiona hambrunas y desnutrición (Sánchez et al., 2009; Más, 2010).

\section{Contaminación atmosférica.}

Incremento en el aire de gases y partículas en suspensión, como aerosoles sólidos o líquidos.

Problemas en el sistema respiratorio tales como asma agravada, bronquitis crónica, enfermedades cardiopulmonares, disminución de la función pulmonar y cáncer del aparato respiratorio (Feo, 2009).

En los ecosistemas con climas más áridos y secos, o los de aire caliente y húmedo, pueden presentar y empeorar la contaminación del aire con material particulado de diferente granulometría que puede incluso provocar tormentas de polvo y disminuir la visibilidad.

4. Afectación de la calidad del suelo, reducción de la producción agrícola y la pesca

Posibles migraciones de población y potenciales conflictos sociales por el agotamiento de recursos de por sí ya escasos como el agua, los suelos fértiles y la pesca (IPCC, 2007; Thuiller, 2007; Peterson et al., 2010). 
Afectación de la nutrición y seguridad alimentaria (Avilez, Bazalar, Azañedo y Miranda, 2016), en particular para aquellas poblaciones que dependan más de estas actividades (Sánchez, 2016).

La reducción las reservas de agua dulce y la menor disponibilidad de agua potable, fuerza a las personas a utilizar recursos de agua dulce de menor calidad, a menudo contaminados por agentes patógenos o sustancias químicas que incrementan la incidencia de diarreas $\mathrm{y}$ otros trastornos gastrointestinales.

\section{Evento - Efectos}

4. Afectación de la calidad del suelo, reducción de la producción agrícola y la pesca La reducción de agua disponible para la higiene personal produce infecciones de la piel y empeora la eficiencia de los sistemas de desagüe, lo cual a su vez provoca mayores concentraciones de desechos contaminantes y patógenos en las reservas de agua sin tratar (Feo, 2009).

5. Cáncer y enfermedades crónicas degenerativas

Favorecidos por factores como los contaminantes ambientales, las radiaciones solares intensas que atraviesan la cada vez más delgada capa de ozono atmosférica, los productos químicos empleados en la producción de alimentos, la mala calidad del agua (Veliz y Bianchetti, 2013; Feo, 2009).

\section{Emergencia y reemergencia de enfermeda- des infecciosas}

Enfermedades emergentes: causadas por nuevos agentes o por factores causales ya conocidos que recientemente han adquirido un carácter epidémico, por convertirse en una amenaza en las regiones en las que antes no existían, por ejemplo: virus zika, encefalitis del virus nipah, síndrome agudo respiratorio severo [SARS] e influenza aviar H5N1.

Enfermedades reemergentes: enfermedades previamente descritas, controladas o tratadas eficazmente, incluso erradicadas en algunas áreas y cuya frecuencia se encuentra en aumento porque los agentes manifiestan resistencia a medicamentos, tienen formas más virulentas y distribución geográfica generalizada, por ejemplo: tuberculosis, malaria, dengue, la fiebre chikungunya, la enfermedad de Lyme y el virus del oeste del Nilo) (Soler, Hernández, Pabón y Tenjo, 2013; Kuri, Guzmán, De La Paz y Salas, 2015).

Pueden ser: Antroponosis: la especie humana es el hospedero primario y generalmente exclusivo; por ejemplo, el virus de inmunodeficiencia humana/sida, el sarampión, la malaria, el dengue y la fiebre amarilla.

Zoonosis: las especies animales son el reservorio natural de los agentes infecciosos; por ejemplo: la rabia, la peste bubónica y la enfermedad de Lyme.

\subsection{Enfermedades transmitidas por vec- tores}

Diversos factores propician las condiciones para que los distintos reservorios y vectores de enfermedades transmisibles, entre los cuales se destacan roedores, mosquitos y garrapatas (Gorodner, Martino, Neira y Besuschio, 2013) puedan ampliar las poblaciones, su potencial propagación y el rango de acción geográfico a zonas (aparecen en latitudes y altitudes mayores), donde encuentra un alto número de personas susceptibles (Medina, 2010; Menghi, 2007; Cerda, Valdivia, Valenzuela y Venegas, 2008; Gonzales et al., 2014). 
Hay una relación significativamente positiva entre las variaciones de las precipitaciones, la temperatura y la humedad, al crear condiciones beneficiosas para el ciclo de vida y el desarrollo de los agentes patógenos o de los vectores que se reproducen en medios acuáticos (OMS, 2003; Sutherst, 2004; Lozano, 2016), al convertirlos en infecciosos más rápidamente (por ejemplo, disminuye el periodo de maduración del Plasmodium), se aumenta la longevidad del mosquito anófeles (Iniesta et al., 2008; Lemus y Corratgé, 2009), al tiempo que algunos géneros de mosquitos vectores se multiplican exponencialmente, al incrementarse la tasa de producción de huevos; por ejemplo, hay una mayor incidencia del dengue (Rifakis et al, 2005), pero este puede presentarse incluso durante periodos con bajas precipitaciones, por el hábito de acumular agua en contenedores que se convierten en criaderos del vector (OMS, 2003; Sutherst, 2004).

\section{Conclusiones}

Los fenómenos secundarios al cambio climático vienen generando impactos negativos diversos, directos e indirectos en la epidemiología de las enfermedades e inequidades en la salud de las personas.

Estas repercusiones varían según escalas espaciales y temporales y dependen de condiciones socioeconómicas y ambientales cambiantes, tales como la composición atmosférica, la urbanización, el desarrollo económico y social, el comercio internacional, las migraciones humanas, el desarrollo industrial y el uso de la tierra. No obstante, muchas de estas afectaciones se solapan unas con otras, por lo que son difícilmente reversibles en años o de- cenios, al tiempo que algunas de ellas son evitables, mitigables o controlables.

Las investigaciones realizadas en torno a los efectos del cambio climático se han enfocado en las repercusiones sobre los ecosistemas y las interacciones de los fenómenos climáticos sobre la dinámica de la flora y fauna silvestre. De igual modo, se ha estudiado más las enfermedades infecciosas zoonóticas, debido a la interacción más frecuente entre el ser humano y la fauna silvestre, durante el desarrollo de actividades económicas sustentadas en la explotación de recursos naturales en ecosistemas no intervenidos.

La mayoría de los estudios sobre las afectaciones del cambio climático a la salud de las personas han centrado su atención en escenarios de un planeta con tendencia creciente al calentamiento global, pero, en general, en ellos no se describen enfermedades asociadas a un enfriamiento del planeta, al punto que se considera que el aumento de la temperatura terrestre trae beneficios en las zonas de temperaturas extremas frías, dado que los inviernos más suaves reducirían el pico invernal de mortalidad en estos países.

Desde la salud pública se puede y se debe participar activamente en la definición e implementación de políticas y acciones de investigación en la identificación, comprensión, evaluación y prevención (reducir los daños) de los posibles riesgos o efectos para la salud humana, dado que las influencias del clima en la salud se ven moduladas a menudo por determinantes no climáticos, como las interacciones de las comunidades con otros procesos ecológicos y sus condiciones sociales y políticas de adaptación. 


\section{REFERENCIAS BIBLIOGRÁFICAS:}

Avilez, J., Bazalar,. J, Azañedo, D. Y MiRANDA, J.

(2016) Perú, cambio climático y enfermedades no trasmisibles: ¿dónde estamos y a dónde vamos?. Revista Perú Medicina Experimental Salud Pública, 33(1), 143-148. Recuperado de http://www.rpmesp.ins. gob.pe/index.php/rpmesp/article/ view/2016

BALlester, J. y MOReno, J.

(2006) Cambio climático y salud pública: escenarios después de la entrada en vigor del Protocolo de Kioto. Gaceta Sanitaria, 20(Supl 1), 160-174. Recuperado de http://www.sciencedirect. com/science/article/pii/ S0213911106715801

Beniston. M.

(2002) Climatic change: possible impacts on human health. Swiss Medical Weekly, (132), 332-337. Recuperado de http://www.unige.ch/climate/ Publications/Beniston/SMW02.pdf

Berberian, G. y RosanovaA, M.

(2012) Impacto del cambio climático en las enfermedades infecciosas. Archivos Argentinos de Pediatría, 110(1), 39-45. Recuperado de Http://www. slipe.org/artsocios/Cambio\%20 climatico.pdf

Burstein, T.

(2016) Rol del sector salud ante el cambio climático. Revista Peruana de Medicina Experimental y Salud Públi- ca, 33(1), 139-142. doi:10.17843/ rpmesp.2016.331.2015. Recuperado de http://www. scielosp.org/ $\mathrm{pdf} / \mathrm{rpmesp} / \mathrm{v} 33 \mathrm{n} 1 / 1726-4642-\mathrm{rp}-$ mesp-33-01-00139.pdf

Butler, D. y Harley, D.

(2010) Primary, secondary and tertiary effects of eco-climatic change: the medical response. Postgraduate Medical Journal, (86), 230-234. doi:10.1136/pgmj.2009.082727. Recuperado de http://pmj.bmj.com/ content/86/1014/230.long

Butler, D., Corvalan, C. y Hillel, K.

(2005) Human health, well-being, and global ecological scenarios. Ecosystems, 8(2), 153-162. Recuperado de http://link.springer.com/article/10.1007/s10021-004-0076-0

Calvo, E.

(2008) Cambio climático y salud humana: un mensaje reiterado desde 1995. Revista Peruana de Medicina Experimental y Salud Pública, 25(4), 410-412. Recuperado de http:// www.scielo.org.pe/pdf/ rins/v25n4/ a11v25n4

Cerda, J., Valdivia, G., Valenzuela, M. Y VENEGAS, J.

(2008) Cambio climático y enfermedades infecciosas. Un nuevo escenario epidemiológico. Revista Chilena de Infectología, 25(6), 447-452. Recuperado de http:// www.scielo.cl/pdf/ rci/v25n6/art06.pdf 
Convención Marco de las Naciones Unidas sobre el Cambio Climático, Naciones UNIDAS.

Recuperado de https://unfccc.int/ files/essential_background/background_publications_htmlpdf/ application/ pdf/convsp.pdf

Costello, A., Abbas, M., Allen, A., Ball, S., Bell, S., Bellamy, R., Craig, P. et al.

(2006) Managing the health effects of climate change. Recuperado de https://www.ucl.ac.uk/global-health/ project-pages/lancet1/ucl-lancetclimate- change.pdf

Feo, O., Solano, E., Beingolea, L., Aparicio, M., Villagra, M., Prieto, M. y Silveti, R.

(2009) Cambio climático y salud en la región andina. Revista Peruana de Medicina Experimental y Salud Pública, 26(1), 83-93. Recuperado de http://www.scielo.org.pe/pdf/rins/ v26n1/a16v26n1.pdf

Gonzales G., Zevallos, A., Gonzales, C., Núñez, D., Gastañaga, C., Cabezas, C. Y STEENLAND, K.

(2014) Contaminación ambiental, variabilidad climática y cambio climático: una revisión del impacto en la salud de la población peruana. Revista Peruana de Medicina Experimental y Salud Pública, 31(3). Recuperado de http://www.scielo.org.pe/scielo.php?pid=S1726 46342014000300021 \&script $=$ sci_ arttext\&tlng=en

Gorodner, J., Martino, A., Neira, J. y BesUSCHIO, C.

(2013) Cambio climático y salud humana. Revista de la Asociación Médica
Argentina, 126(1). Recuperado de https://www.ama-med.org.ar/descargacontenido/5

Haines, A. y Patz, J.

(2004) Health effects of climate change. JAMA, 291(1), 99-103. doi:10.1001/ jama.291.1.99. Recuperado de http://jama.jamanetwork.com/article.aspx?Articleid=197911

Hijar, G., Bonilla, C., Munayco, C., GutiÉRREZ, E. Y RAMOS, W.

(2016) Fenómeno el niño y desastres naturales: intervenciones en salud pública para la preparación y respuesta. Revista Peruana de Medicina Experimental y Salud Pública, 33(2), 300-310. doi:10.17843/rpmesp.2016.332.2205

Huynen, M., Martens, P., Schram, D., WeiJenberg, M. y Kunst, A.

(2001) The impact of heat waves and cold spells on mortality rates in the Dutch population. Environmental Health Perspectives, 109(5). Recuperado de http://www.ncbi.nlm.nih. gov/pmc/articles/PMC1240305/

Iniesta, N., Ríos, J., Fernández, M. y BARBADO, F.

(2009) Cambio climático: ¿nuevas enfermedades para un nuevo clima? Revista Clínica Española, 209(5), 234240. Recuperado de http://www. sciencedirect.com/ science/article/ pii/S0014256509712408

JimÉNEZ, C. Y SOLEDAD, J.

(2001) Los desplazados ambientales, más allá del cambio climático. Un debate abierto. Cuadernos Geográfi- 
cos, 49(2), 201-215. Recuperado de http://revistaseug.ugr.es/index.php/ cuadgeo/ article/view/571/659

Kuri, P., Guzmán, E., De la Paz, E. y Salas, A.

(2015) Enfermedades emergentes y reemergentes. Gaceta Médica de México, (151), 674-680. Recuperado de http://www.medigraphic.com/pdfs/ gaceta/gm-2015/gm155q.pdf

Ledrans, M., Pirard, P., Tillaut, H., Pascal, M., Vandentorren, S., Suzan, F. y Delmas, M.

(2004) The heat wave of August 2003: what happened? Recuperado de http://www.ncbi.nlm.nih.gov/ pubmed/15461047

Lemus, E. y Corratgé, H.

(2009) Cambio climático y dengue en Cuba. Revista Cubana de Medicina General Integral, 25(4), 196-207. Recuperado de Http://www.bvs.sld.cu/revistas/ mgi/vol_25_4_09/mgi19409.htm

\section{LOZANO, J.}

(2016) La actuación femenina en la adaptación al cambio climático en el espacio urbano. Un estudio de caso en la Amazonía peruana. Encrucijadas. Revista Crítica de Ciencias Sociales, 11(1), 1-26. Recuperado de http:// www.encrucijadas.org/index.php/ ojs/article/view/257/207

Más, B. P.

(2010) El cambio climático: un reto para los sistemas de salud. Revista Cubana de Higiene y Epidemiología, 48(3), 226-228. Recuperado de http://scielo.sld.cu/pdf/hie/v48n3/hie01310. pdf
McMichael, A.

(2003) Global climate change and health: an old story writ large. Chapter one. En A. J.

McMichael, D. H.

Campbell-Lendrum, C. F. Corvalan, K. L. Ebi, A. Githeko, J. D. Scheraga et al., Climate change and human health: risks and responses (pp. 1-17). Ginebra: World Health Organization. Recuperado de http://www.who.int/globalchange/ publications/climchange.pdf

McMichael, A.

(2014) Earth as humans' habitat: global climate change and the health of populations. International Journal of Health Policy and Management, 2(1), 9-12. Recuperado de http:// doi. org/10.15171/ijhpm.2014.03

Medina, G.

(2010) Ecología de enfermedades infecciosas emergentes y conservación de especies silvestres. Archivos de Medicina Veterinaria, (42), 11-24. Recuperado de http://www.scielo. cl/pdf/amv/v42n1/art03.pdf

Mendoza, H., Martínez, J., Contreras, E. Y FAJARDO, M.

(2012) Influencia del calentamiento global en la salud humana. Recuperado de http://www.medigraphic.com/pdfs/ revcubtecsal/cts-2011/cts112h.pdf

Menghi, C.

(2007) Calentamiento global: el riesgo oculto para la salud. Revista Argentina de Microbiología, 39(3), 131- 
132. Recuperado de http://www.redalyc.org/pdf/2130/213016790001. pdf

National Institute of Health.

(NIH, 2010). A human health perspective on climate change a report outlining the research needs on the human health effects of climate change. The Interagency Working Group on Climate Change and Health. Recuperado de https://www.niehs.nih.gov/ health/materials/a_human_health_ perspective_on_climate_change_ full_report_508.pdf. https://www. ipcc.ch/pdf/assessment-report/ ar5/ wg2/drafts/fd/WGIIAR5-Chap11_ FGDall.pdf

Noyes, P., Mcelwee, M., Miller, H. Clark, B., Van Tiem, L., Walcott, K., Erwin, K. y LeVIN, E.

(2009) The toxicology of climate change: Environmental contaminants in a warming world. Environment International, 35(6), 971-986. Recuperado de http://www.sciencedirect.com/science/article/pii/ S0160412009000543

Ochoa, M., Castellanos, R., Ochoa, Z. Y Oliveros, J.

(2015) Variabilidad y cambio climáticos: su repercusión en la salud. Medisan, 19(7), 873-885. Recuperado de http://scielo.sld.cu/ scielo.php?script=sci_ arttext\&pi$\mathrm{d}=$ S1029-30192015000700008\&ln$\mathrm{g}=\mathrm{es} \& \operatorname{tlng}=\mathrm{es}$

Orellana, N. y Navarro, D.

(2010) Los cambios climáticos y su implicación en la salud. Revista Agua
Ambiente. Recuperado de http:// www.portalces.org/sites/default/files/migrated/docs/AGUA_Y_CC_ BOLIVIA. pdf $\#$ page $=63$

OrganizaCión INTERNACIONAL PARA LAS Migraciones.

(OIM, 2007). Nonagésima cuarta reunión. Nota para las deliberaciones: la migración y el medio ambiente. MC/INF/288. Recuperado de https://www.iom.int/ jahia/webdav/ site/myjahiasite/shared/shared/ mainsite/microsites/IDM/workshops/evolve ng_global_ economy_2728112007/MC_INF_288_ES.pdf

\section{Organización Mundial de la Salud.}

(OMS, 2003). Cambio climático y salud humana: riesgos y respuestas: Resumen. Recuperado de http://apps.who.int/ iris/bitstream/10665/42808/1/9243590812. pdf

\section{Organización Mundial de la Salud.}

(OMS, 2008). Protección de la salud frente al cambio climático día mundial de la salud 2008. Resumen de un documento temático. Recuperado de http://www.who.int/world-healthday/ toolkit/report_web_summary_es_whd.pdf?Ua=1

\section{Organización Mundial de la Salud.}

(OMS, 2016a). Cambio climático y salud. Ginebra: OMS. Recuperado de http:// www.who.int/mediacentre/factsheets/fs266/es/

\section{Organización Mundial de la Salud.}

(OMS,2016b).Género,cambioclimáticoysalud. Recuperado de http://apps. who.int/ 
iris/bitstream/10665/204178/1/9789243508184_spa.pdf

Panel Intergubernamental de Expertos Sobre el CAMbio Climático

(IPCC, 2001) Tercer informe de evaluación cambio climático 2001. La base científica. Parte de la contribución del Grupo de trabajo I al Tercer Informe de Evaluación Grupo Intergubernamental de Expertos sobre el Cambio Climático. Recuperado de https:// www.ipcc.ch/pdf/climate-changes-2001/synthesis-syr/spanish/wg1-summaries.pdf

Panel Intergubernamental de Expertos sobre el CAmbio Climático.

(IPCC, 2007). Cambio climático 2007: Informe de síntesis. Cuarto Informe de evaluación del Grupo Intergubernamental de Expertos sobre el Cambio Climático. Recuperado de https:// www.ipcc.ch/pdf/assessment-report/ar4/syr/ar4_syr_sp.pdf

Panel Intergubernamental de Expertos Sobre el CAMbio Climático.

(IPCC, 2014). Cambio climático 2014: Impactos, adaptación y vulnerabilidad. Resumen para responsables de políticas. Contribución del Grupo de trabajo II al Quinto Informe de Evaluación (GTII IE5) del Grupo Intergubernamental de Expertos sobre el Cambio Climático (IPCC). Recuperado de https://www.ipcc. ch/pdf/assessment-report/ar5/wg2/ ar5_wgII_spm_es.pdf

Patz, J., Graczykb, T., Gellera, N. y Vittor, A.

(2000) Effects of environmental change on emerging parasitic diseases. In- ternational Journal for Parasitology. Recuperado de http://citeseerx. ist.psu.edu/viewdoc/dow nload?$\mathrm{doi}=10.1 .1 .525 .9839 \& \mathrm{rep}=\mathrm{rep} 1 \&-$ type $=$ pdf

Patz, J., Mcgeehin, M., Klebi, B., Epstein, P., Grambsch, A., Gubler, D. Trtanj, J. et Al.

(2000) The potential health impacts of climate variability and change for the United States: executive summary of the report of the health sector of the U.S. National Assessment. Environmental Health Perspectives, 108(4), 367- 376. Recuperado de http://www.ncbi.nlm.nih.gov/pmc/ articles/PMC1638004/

Petersson, R., Marrero, M. y Taboada, C.

(2010) Cambio climático y salud humana. Revista Médica Electrónica, 32(4). Recuperado de http://www. revmatanzas.sld.cu/revista $\% 20 \mathrm{me}$ dica/ano\%202010/vol4\%202010/ tema09.htm

Ponce, Y. y Cantú, P.

(2012) Cambio climático: bases científicas y escepticismo. CULCyT, 9(46). Recuperado de http://erevistas.uacj. $\mathrm{mx} /$ ojs/index.php/culcyt/article/ view/174/168

Rifakis, P., Gonçalves, N., OMaña, W., HerNÁndeZ, O., Manso, M., Intingaro, R. y RoDRÍGUEZ, A.

(2005) Asociación entre las variaciones climáticas y los casos de dengue en un hospital de Caracas, Venezuela, 1998-2004. Revista Peruana de Medicina Experimental y Salud Pública, 22(3). Recuperado de http:// www.scielo.org.pe/scielo.php?pi- 
$\underline{\mathrm{d}=S 1726-46342005000300005 \& \mathrm{~s}-}$ cript=sci_arttext

SÁNCHEZ, C.

(2016) Evolución del concepto de cambio climático y su impacto en la salud pública del Perú. Revista Peruana de Medicina Experimental y Salud Pública, 33(1), 128-138. doi:10.17843/ rpmesp.2016.331.2014. Recuperado de http://www.scielosp.org/ $\mathrm{pdf} / \mathrm{rpmesp} / \mathrm{v} 33 \mathrm{n} 1 / 1726-4642-\mathrm{pp}-$ mesp-33-01-00128.pdf

SÁnchez, L., Mattar, S. y GonzÁlez, M.

(2009) Cambios climáticos y enfermedades infecciosas: nuevos retos epidemiológicos. Revista MVZ Córdoba, 14(3), 1876-1885. Recuperado de http://www.scielo.org.co/ pdf/mvz/ v14n3/v14n3a12.pdf

Soler, D., Hernández, P., Pabón, L. y Tenjo, A.

(2013) Pérdida de biodiversidad: un factor determinante en el aumento de enfermedades infecciosas compartidas entre humanos y animales. Revista Biodiversidad Colombia, (2). Recuperado de http://revistas. lasalle.edu.co/index.php/bi/article/ view/2743/2331
Sutherst, R.

(2004) Global change and human vulnerability to vector-borne diseases. Clinical Microbiology Reviews, 17(1), 136-173. Recuperado de http:// www.ncbi.nlm.nih.gov/pmc/articles/PMC321469/ pdf/0089.pdf

Thuiller, W.

(2007) Climate change and the ecologist. Revista Nature, 448(2). Recuperado de http://www. nature.com/nature/ journal/v448/n7153/pdf/448550a. pdf

UsERos, J.

(2014) El cambio climático y sus efectos sobre la salud humana. Anales de la Real Academia de Medicina y Cirugía de Valladolid, (51), 23-54. Recuperado de https://dialnet.unirioja. es/descarga/articulo/5361603.pdf

Veliz, L. y BiAnChetti, A.

(2013) Cambio climático y salud pública: acciones desde la institucionalidad en el escenario sociocultural actual. Revista Costarricense de Salud Pública, 22(2). Recuperado de http:// www. scielo.sa.cr/pdf/rcsp/v22n2/ $\operatorname{art12} \mathrm{v} 22 \mathrm{n} 2$.pdf 
